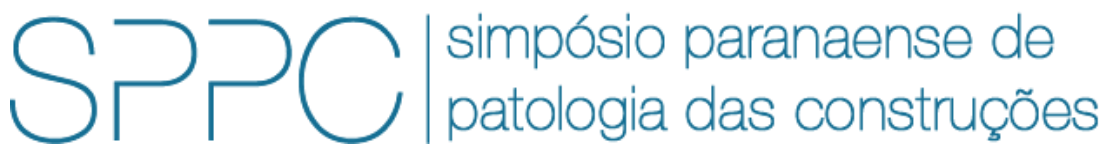

ISSN 2526-7248 artigo n. 2SPPC2004, pp. 500-511, 2017

\title{
Estudo da influência da compactação de base no surgimento de trincas em pisos industriais de concreto considerando a interação solo estrutura
}

\author{
Gabriel Caviglione ${ }^{1}$ e Jeselay dos Reis ${ }^{2}$ \\ ${ }^{1}$ Mestrando, Eng. Civil, Universidade Estadual de Maringá, gtcaviglione@gmail.com \\ 2Prof. Associado, Universidade Estadual de Maringá, jeselay@hotmail.com
}

Resumo: Pisos industriais de concreto consistem em estruturas de múltiplas camadas destinadas a transferir os esforços do tráfego de pessoas e equipamentos para o maciço de fundações (subleito), garantindo segurança e conforto. As condições de execução dessas camadas refletem no desempenho do piso, especialmente em suas características mecânicas. Compactações não regulares provocam não uniformidade nos valores do módulo de reação do solo $\left(k_{v}\right)$, causando aumento de momentos fletores que podem provocar fissuração da peça. Avaliou-se a influência de baixos valores de módulos de reação sobre o piso de concreto, em faixas localizadas. Diversas posições da faixa de $k_{v}$ reduzido e diferentes valores de rigidez relativa foram analisados por meio do método dos elementos finitos. Os modelos numéricos mostraram que a introdução da faixa de $k_{v}$ reduzido provocou inversão de momentos fletores para todas as espessuras de piso. Os modelos mais rígidos foram menos influenciados, recomenda-se além da compactação rigorosa, a adoção de uma rigidez mínima em projeto. A posição mais crítica depende da rigidez da placa, ao contrário da posição de menor influência, que foi a $20 \%$ do comprimento. Os maiores momentos adicionais foram da ordem de $12 \mathrm{kNm} / \mathrm{m}$.

Palavras-chave: Modelo de Winkler, Piso Industrial de Concreto, Patologia.

Abstract: Industrial concrete floor are composed by multi-layered structures developed to lead traffic load, of people and equipment, to the foundation (subgrade), with security and comfort. Execution conditions of these layers will influence slab's performance, especially on its mechanical characteristics. Non regularities on compactions will provoke non uniformity on soil's reaction module $\left(k_{v}\right)$, increasing on flexural moments that can induce cracking on the slab. Was assessed the influence of low soil's reaction value on concrete slab floors, in located regions. Several positions of reduced $k_{v}$ region's and several relative stiffness values were analysed through finite element method. Numerical models have shown that the introduction of the reduced $k_{v}$ region provoked inversion of flexural moments, on all slab thickness. But models with greater stiffness have presented less influence to these effects, so it's recommended to design concrete slab floor with a minimal stiffness, besides the rigorous compaction control. Critical region can't be determined, because it's dependent on relative stiffness, although region's position that has less influence on slabs behavior was at $20 \%$ of length. The greatest increase on flexural moments were about $12 \mathrm{kNm} / \mathrm{m}$.

Keywords: Winkler Model, Industrial Concrete Floor, Pathology. 


\section{Introdução}

Os pavimentos são formados por estruturas de múltiplas camadas responsáveis por transferir os esforços do tráfego ao maciço de solos. Os pavimentos rígidos de concreto e são empregados para grandes esforços e em pisos industriais. Estes pavimentos são compostos com uma camada de concreto armado apoiada em uma base de brita graduada simples, esta brita é executada sobre uma sub-base de solo ou diretamente sobre subleito.

Deficiências na sub-base ou no subleito ocasionam falhas mecânicas no piso de concreto. Estas falhas mecânicas vão resultar em trincas e rachaduras no piso de concreto, semelhante às trincas da Figura 1.

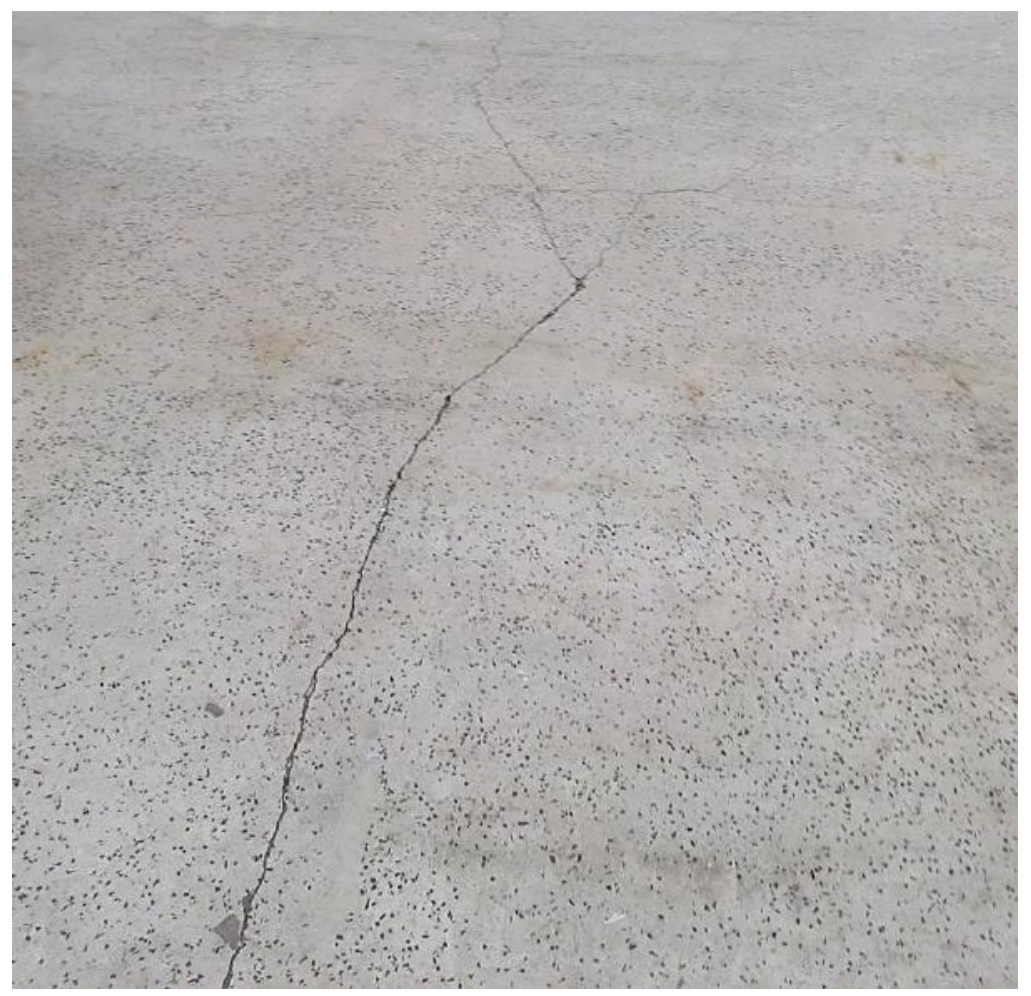

Figura 1: Trincas em galpão logístico em Maringá-PR

Por isso, se faz importante estudar a contribuição de deficiências na compactação da base do piso para o surgimento de trincas. Foi simulada uma variação no módulo de reação do solo, a fim de se inferir sobre esforços e aparecimento de trincas no piso. Espera-se estabelecer regiões críticas para o controle da compactação além de investigar a cerca da influência da rigidez do piso sobre os recalques e esforços.

\section{Pisos Industriais}

Define-se piso industrial como sistema de múltiplas camadas com finalidade de resistir e distribuir os esforços verticais provenientes dos carregamentos ao subleito, conforme a ANAPRE (Associação Nacional de Pisos e Revestimentos de Alto Desempenho) [5]. De maneira geral, os pisos são compostos por: uma sub-base, com função de regularizar e melhorar a capacidade de suporte; uma lona plástica (geralmente polietileno), a fim de impedir ascensão de umidade e reduzir o atrito; o 
CAVIGLIONE, G.; REIS, J., ESTUDO DA INFLUÊNCIA DA COMPACTAÇÃO DE BASE NO SURGIMENTO DE TRINCAS EM PISOS INDUSTRIAIS DE CONCRETO CONSIDERANDO A INTERAÇÃO SOLO ESTRUTURA. $2^{\circ}$ Simpósio Paranaense de Patologia das Construções (2० SPPC), artigo 2SPPC2004, pp. 500-511, 2017. DOI: 10.4322/2SPPC.2017.041

elemento de concreto, que apresenta função estrutural e por isso é armado [1,3]. A Figura 2 mostra estrutura de um piso industrial.

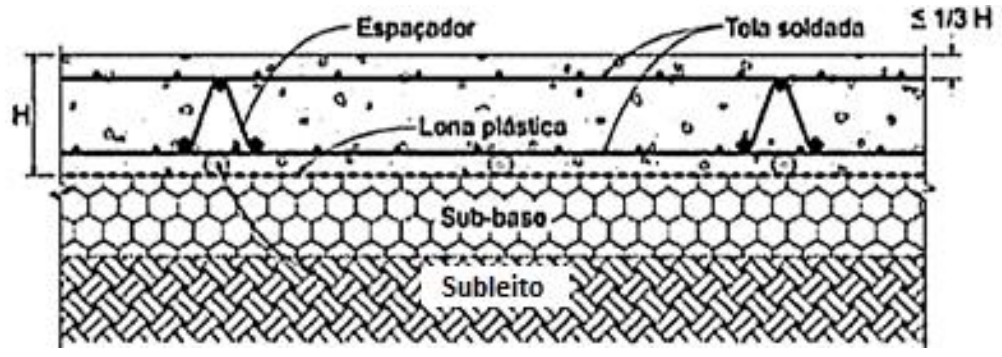

Figura 2: Corte transversal típico em piso de concreto [1].

Os pisos de concreto funcionam como um radier apoiado sobre o solo. Velloso e Lopes [6] registram que as cargas são transmitidas ao solo através de pressões de contato que dependem da rigidez da fundação, do tipo de solo, das características e intensidade das cargas. A Figura 3 ilustra as pressões mobilizadas no contato de placas com o solo.

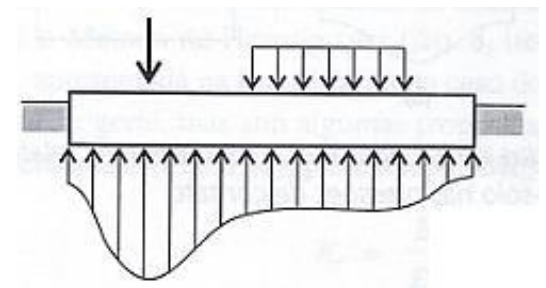

Figura 3: Distribuição de pressões de contato, conforme a carga [6].

A Figura 4 ilustra a distribuição das pressões de contato para solos arenosos e argilosos. Nas argilas o efeito da coesão concentra tensões nas bordas, pois os elementos de solo vizinhos à sapata/radier também são solicitados. Em areias os recalques são maiores nas bordas por causa do confinamento do centro, de tal forma que sapatas/radier rígidas aplicam maiores pressões no centro, para produzirem mesmo nível de deformação [7].

A1)

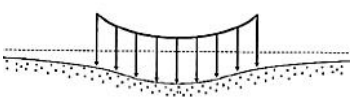

A2)

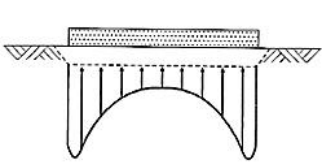

B1)

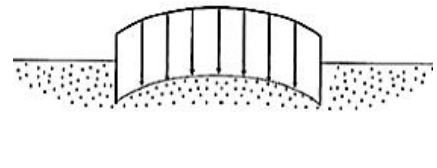

B2)

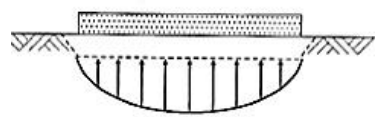

Figura 4: Distribuição de pressões de contato no solo, A - refere-se a argilas, B - a areias, 1 - estruturas flexíveis, 2 - rígidas. Adaptado de [7].

Simular as pressões de contato não é uma tarefa simples. Dutta e Roy [9] apresentam diversas maneiras de proceder com a simulação, neste trabalho será usado o modelo de Winkler. Este consiste em representar o comportamento reológico do maciço de solo como uma associação de molas elásticas, de variação linear e independentes [9]. A Figura 5 representa o modelo de Winkler. 


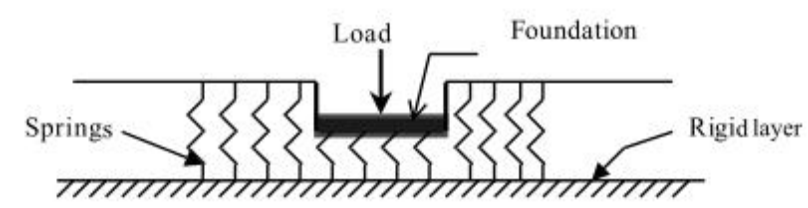

Figura 5: Modelo de Winkler [9].

O conceito do módulo de reação é expresso pela equação 1 , relaciona 0 deslocamento (recalque) com a tensão aplicada. É importante ressaltar que a interpretação física do módulo é limitada, pois depende das condições geométricas da placa e das condições mecânicas do solo [9].

$$
k_{v}=\frac{\sigma}{\rho}
$$

Onde: $k_{v}$ é o módulo de reação vertical do solo

$\sigma$ é a tensão atuante no solo

$\rho$ é o recalque do elemento de fundação

Devido sua praticidade, o modelo de Winkler tem sido amplamente empregado para simular placas apoiadas sobre o solo [2,9]. Dutta e Roy [9] apresentam uma crítica aos diversos modelos utilizados no estudo da interação solo-estrutura, registrando que a maior dificuldade do modelo de Winkler é ausência de continuidade do modelo, devido à independência das molas. Além disso, os autores também refletem sobre as dificuldades na determinação do $k_{v}$.

O módulo de reação do solo pode ser obtido por ensaios de placa, tabelas de valores típicos e correlações com o módulo de deformabilidade [8]. Moraes [10] e Cunha [3] ainda trazem tabelas com valores típicos para vários tipos de solo, CBR e módulo de reação, cita-se os ensaios de placa de Terzaghi $[6,11]$. Raush apud Moraes [10] apresenta uma solução para obtenção $k_{v}$ em função do módulo edométrico.

Neste trabalho, o valor do módulo de reação $\left(k_{v}\right)$ foi obtido através do CBR (california bearing ratio). A Figura 6 registra o valor do módulo de reação em função do CBR. Rodrigues et al. [1] também traz valores de $k_{v}$ para bases com melhoramento. 
CAVIGLIONE, G.; REIS, J., ESTUDO DA INFLUÊNCIA DA COMPACTAÇÃO DE BASE NO SURGIMENTO DE TRINCAS EM PISOS INDUSTRIAIS DE CONCRETO CONSIDERANDO A INTERAÇÃO SOLO ESTRUTURA. $2^{\circ}$ Simpósio Paranaense de Patologia das Construções (20 SPPC), artigo 2SPPC2004, pp. 500-511, 2017. DOI: 10.4322/2SPPC.2017.041

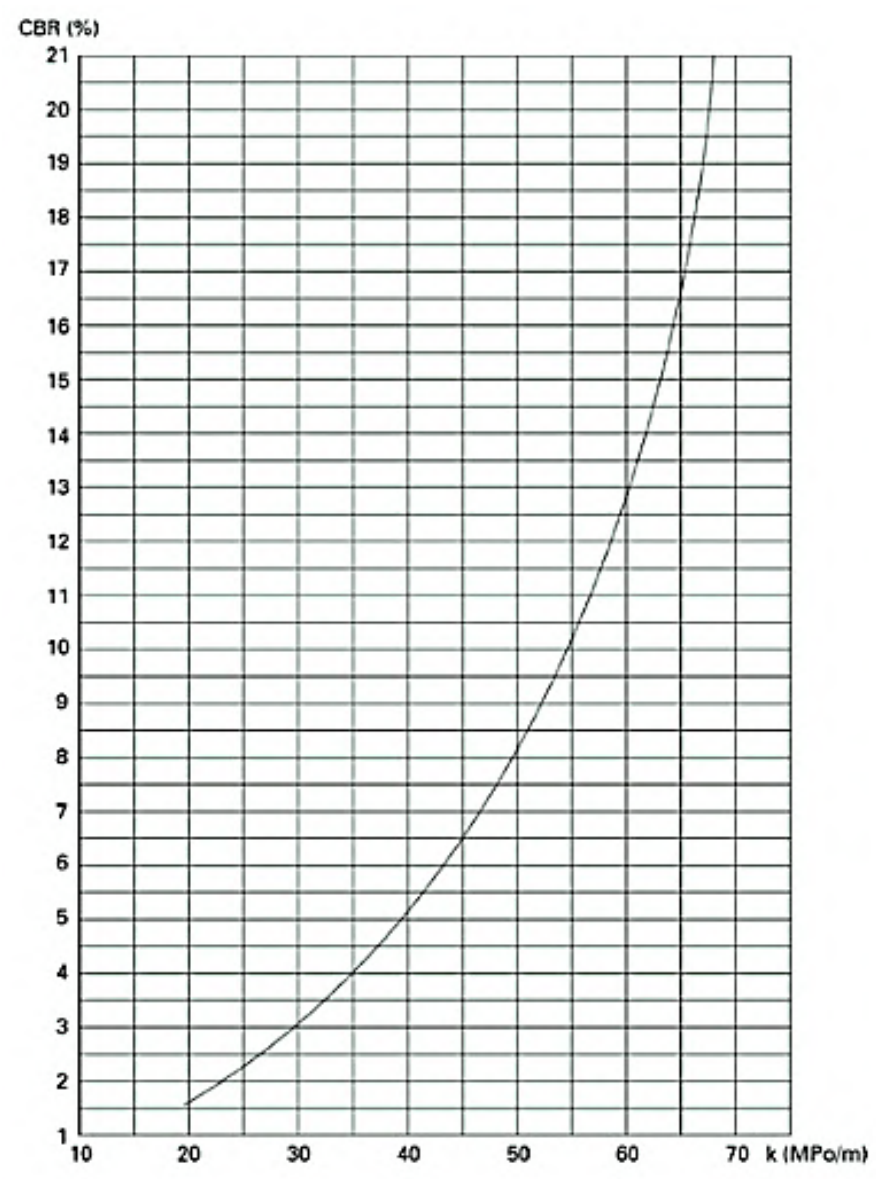

Figura 6: Correlação CBR e Módulo de Reação [4].

Segundo Souza e Reis [8], o comportamento dos pisos depende fundamentalmente da rigidez relativa entre piso e solo, considerada pela análise da ISE (interação solo estrutura). Velloso e Lopes [6] apresentam diversas equações para consideração da rigidez relativa na distribuição das pressões de contato. Destacam-se as expressões de Meyerhof, Schultze e Hetènyi ilustradas nas equações 2, 3 e 6.

$$
K_{s S}=\frac{E_{c} \cdot I}{E_{s} \cdot B^{3}}
$$

Onde: $K_{S S}$ é rigidez relativa solo estrutura

$E_{s}$ é o módulo de deformabilidade do solo

$B$ é a largura da placa

$E_{c}$ é o módulo de elasticidade da estrutura

I é a inércia da placa

$$
K_{S S}=\frac{E_{c} \cdot \frac{t^{3}}{12}}{E_{s} \cdot L^{3}}
$$

Onde:

$t$ é a espessura da placa

$L$ é o comprimento da placa

Souza e Reis [8] observam que se pode obter o módulo de deformabilidade do solo $\left(E_{s}\right)$ por meio da fórmula de Perloff (equação 4), pela teoria da elasticidade.

Onde: $v$ é o coeficiente de Poisson

$$
E_{s}=k_{v}\left(1-v^{2}\right) \cdot B \cdot I_{w} \text {. }
$$


$I_{w}$ é um fator de influência

A equação diferencial que resolve o problema de placas sobre apoios elásticos é apresentada na equação 5 [12]. Esta equação pode apresentar solução analítica ou numérica.

$$
\frac{\partial^{4} \omega}{\partial x^{4}}+2 \frac{\partial^{4} \omega}{\partial x^{2} \partial y^{2}}+\frac{\partial^{4} \omega}{\partial y^{4}}=\frac{q(x, y)-k \omega}{D}
$$

Onde: $\omega$ refere-se ao recalque

$D$ é o módulo de rigidez da placa, $D=E_{s} t^{3} / 12\left(1-v^{2}\right)$

$x$ e y referem-se a posição do ponto em estudo

Hetènyi foi um dos primeiros autores a apresentar uma solução analítica para vigas e placas sobre apoios elásticos de Winkler. Este é aplicado em placas com comportamento unidimensional, por ter sido elaborado para vigas. A equação 6 ilustra a rigidez relativa solo-viga segundo o modelo de Hetényi $[6,13]$.

$$
K_{s S}=1 / \lambda ; \lambda=\sqrt[4]{\frac{k_{v} \cdot B}{4 \cdot E_{c} \cdot I}}
$$

Onde: $K_{s S}$ é a rigidez solo estrutura de Hetényi

$$
\begin{aligned}
& \text { Se: } \quad K_{S S}<L / \pi \quad \text { considera-se: rigidez relativa baixa } \\
& L / \pi<K_{S S}<4 L / \pi \quad \text { rigidez relativa média } \\
& K_{S S}>4 L / \pi \quad \text { rigidez relativa elevada }
\end{aligned}
$$

O modelo de Hetènyi foi parametrizado em função do parâmetro $K_{S S}=1 / \lambda$ a fim de se estabelecer comparativos com os outros autores. Neste modelo, as pressões de contato em vigas de rigidez relativa elevada são lineares e essas vigas podem ser resolvidas pela equação 7 [6].

$$
\omega(x)=\frac{2 \cdot R}{B \cdot k_{v} \cdot L} \cdot\left[-3\left(1-2 \frac{a}{L}\right) \frac{x}{L}+\left(2-3 \frac{a}{L}\right)\right]
$$

Onde: $\omega(x)$ é o recalque na posição $x$

$R$ é a força equivalente ao carregamento

$a$ é a posição da força equivalente ao carregamento

O modelo de analítico de dimensionamento mais usado é o de Westergaard, foi desenvolvido apenas para cargas concentradas, aplicadas no centro e na borda de placas circulares, e aplicadas no canto de placas quadradas [1,4]. As formulações de Westergaard apresentam resultados próximos às análises computacionais atuais. As formulações podem ser encontradas em $[1,4]$.

Bowles [13] traz soluções numéricas com emprego do método dos elementos finitos, para vigas e radiers. O método dos elementos finitos consiste na divisão da estrutura em diversos elementos (Figura 7). Para cada elemento é formado um sistema de equações relacionando as ações e rigidez da estrutura. Ao se compatibilizar os sistemas de equações de cada elemento será obtido um único sistema, cuja solução, após aplicação das condições de contorno, representa os deslocamentos e esforços nos pontos iniciais e finais de cada elemento na estrutura. 
O elemento de barra, empregado nas análises, é um elemento com um nó inicial e um nó final. Cada nó está sujeito a deslocamentos $(u, v, w)$ e rotações $\left(\theta_{x}, \theta_{y}, \theta_{z}\right)$ nas três direções. Uma revisão aprofundada, com enfoque em aplicações geotécnicas, pode ser encontrada em Bowles [13].

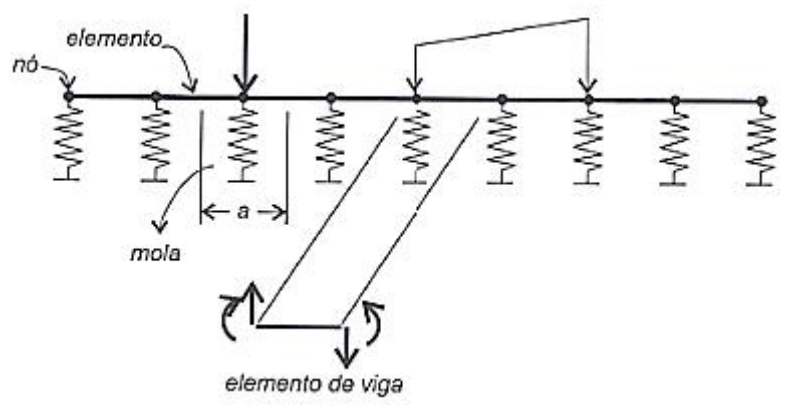

Figura 7: Conceito da solução por elementos finitos. Adaptado de [6].

A principal vantagem do uso do método dos elementos finitos é de se dispor de uma solução computacional para resolver o problema, sendo que diferentemente da solução de Hetènyi, é possível admitir valores diferentes de $k_{v}$ para cada mola.

\section{Metodologia de Análise}

Utilizando-se do método dos elementos finitos pelo software SAP 2000, foram realizadas análises numéricas bidimensionais de elementos lineares. Visando representar placas de $5,00 \times 2,50 \mathrm{~m}$, apoiadas sobre molas espaçadas a cada 25 $\mathrm{cm}$. As placas foram carregadas com uma carga uniforme de $50,0 \mathrm{kN} / \mathrm{m}^{2}$. Nestas condições foram simuladas placas de 10, 15, 20 e $25 \mathrm{~cm}$ de espessura apoiadas sobre solos de $k_{v}=30 \mathrm{MPa} / \mathrm{m}(\mathrm{CBR}=3 \%)$ e $k_{v}=54 \mathrm{MPa} / \mathrm{m}(\mathrm{CBR}=10 \%)$. A análise foi conduzida por unidade de largura da placa. Estes valores baseiam-se nos trabalhos de Cunha [3].

Em uma faixa de 1,50 m (abrangendo 5 molas) foi reduzido o valor do módulo de reação à metade, simulando defeito na compactação ou infiltrações. A variação do valor de $k_{v}$ visa simular as influências na estrutura, e não caracterizar um tipo específico de solo. Cada solo e cada situação diferente irão caracterizar reduções diferentes no $k_{v}$, por isso trata-se de um valor de referência.

A posição da faixa reduzida variou, com passos de $50 \mathrm{~cm}$, do centro para a extremidade, por ser um problema simétrico. Foi desenvolvido um modelo de controle, sem redução do $k_{v}$, para cada espessura. O modelo de controle foi chamado de "A", o modelo com a faixa reduzida centralizada foi de "B" e à medida que a faixa progride para a esquerda refere-se a situação "C", "D" e assim sucessivamente até a situação "F" onde a faixa está na borda. A Figura 8 exemplifica a nomenclatura e as condições mecânicas da análise. 
CAVIGLIONE, G.; REIS, J., ESTUDO DA INFLUÊNCIA DA COMPACTAÇÃO DE BASE NO SURGIMENTO DE TRINCAS EM PISOS INDUSTRIAIS DE CONCRETO CONSIDERANDO A INTERAÇÃO SOLO ESTRUTURA. $2^{\circ}$ Simpósio Paranaense de Patologia das Construções (2० SPPC), artigo 2SPPC2004, pp. 500-511, 2017. DOI: 10.4322/2SPPC.2017.041

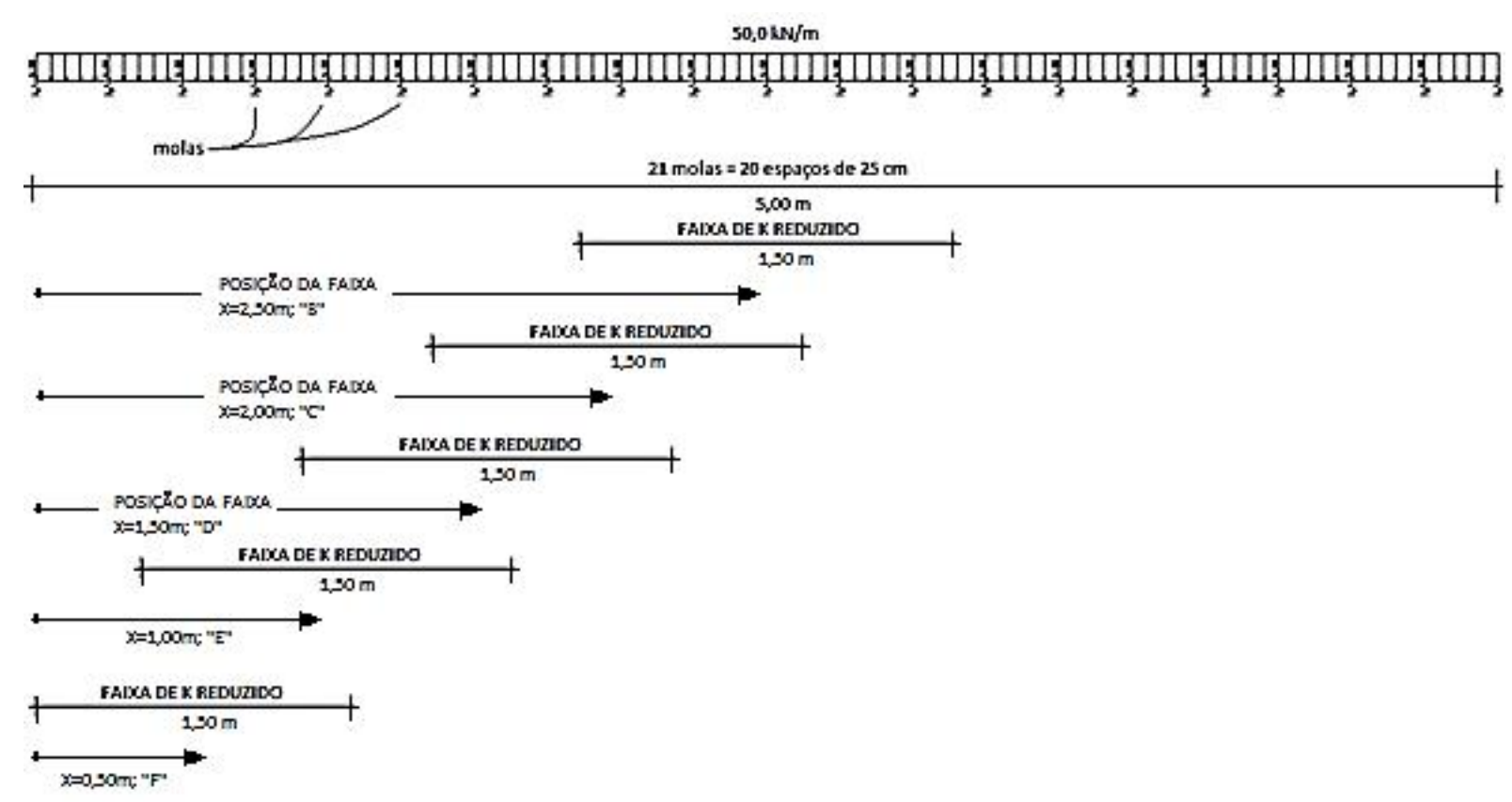

Figura 8: Condições mecânicas da análise e posições da faixa reduzida.

O elemento de barra foi empregado nas simulações, foi desabilitada a análise nas direções que não são pertinentes ao estudo. Ao todo foram elaborados 48 modelos. Os modelos estão nomeados conforme o padrão M10A-CBR3, em que "10" referese à espessura da placa, "A" à situação de carregamento e CBR3 ao CBR de 3\%.

Para as análises referentes à rigidez relativa foram usadas as equações Meyerhof, Schultze e Hetènyi. Para utilizar as equações 2 e 3 se faz necessário o valor do módulo de deformabilidade, obtido pela equação 6 , considerando os valores de $k_{v}$ supramencionados.

\section{Resultados e Análises}

Ao posicionar a faixa no centro da placa, pode-se constatar aumento significativo dos valores de momento positivo em todos os modelos, no entanto, nos modelos mais flexíveis, de espessura igual a $10 \mathrm{~cm}$, houve o surgimento de momentos negativos adjacentes à faixa de redução (Figura 9a).

À medida que a faixa movimenta-se para a borda, esse efeito do surgimento de momentos negativos passa a ocorrer em todos modelos, sendo que em modelos mais flexíveis o momento negativo começa a aparecer antes de a faixa tocar a borda (Figura 9b), e nos modelos mais rígidos o momento negativo só aparece quando a faixa está próxima a extremidade (Figura 9c e 9d). Os maiores valores de momento, em módulo, foram obtidos nos modelos mais rígidos.

a) M10B-CBR3

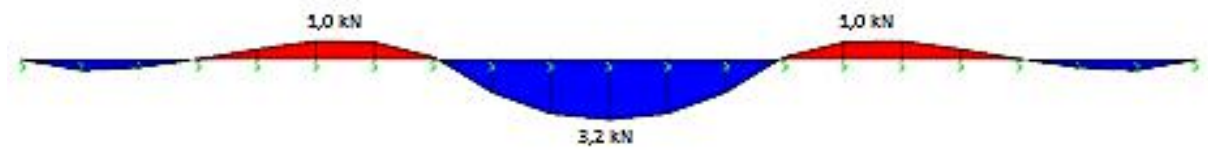


CAVIGLIONE, G.; REIS, J., ESTUDO DA INFLUÊNCIA DA COMPACTAÇÃO DE BASE NO SURGIMENTO DE TRINCAS EM PISOS INDUSTRIAIS DE CONCRETO CONSIDERANDO A INTERAÇÃO SOLO ESTRUTURA. $2^{\circ}$ Simpósio Paranaense de Patologia das Construções (2 SPPC), artigo 2SPPC2004, pp. 500-511, 2017. DOI: 10.4322/2SPPC.2017.041

b) M15C-CBR10

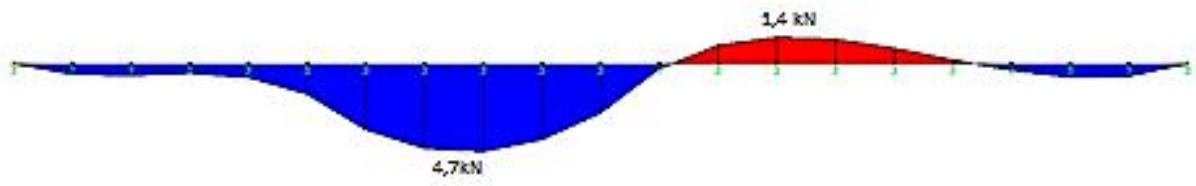

c) M25E-CBR10

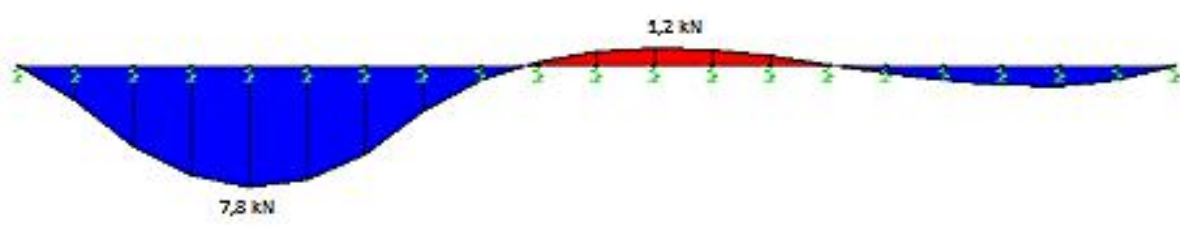

d) M25F-CBR10

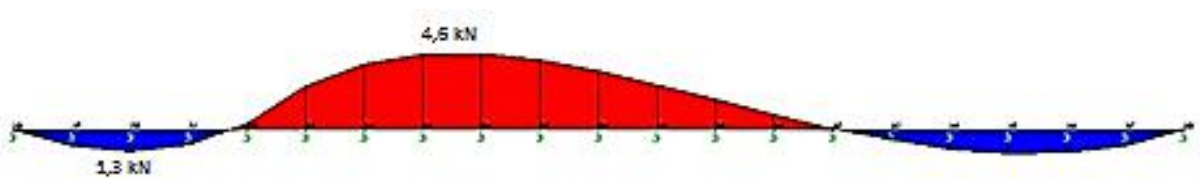

Figura 9: Diagrama de Momento Fletor

A inversão destes momentos gera na estrutura do piso uma solicitação que não estava prevista em projeto, de tal forma que se a armadura mínima e concreto não forem capaz de absorver as trações geradas, será criada uma fissura, como a da Figura 1, causando danos ao pavimento.

A Figura 10 ilustra as deformações do modelo M10A-CBR3, conforme a posição da faixa de $k_{v}$ reduzido. Este comportamento é espelho dos demais modelos, que variam apenas a rigidez relativa.

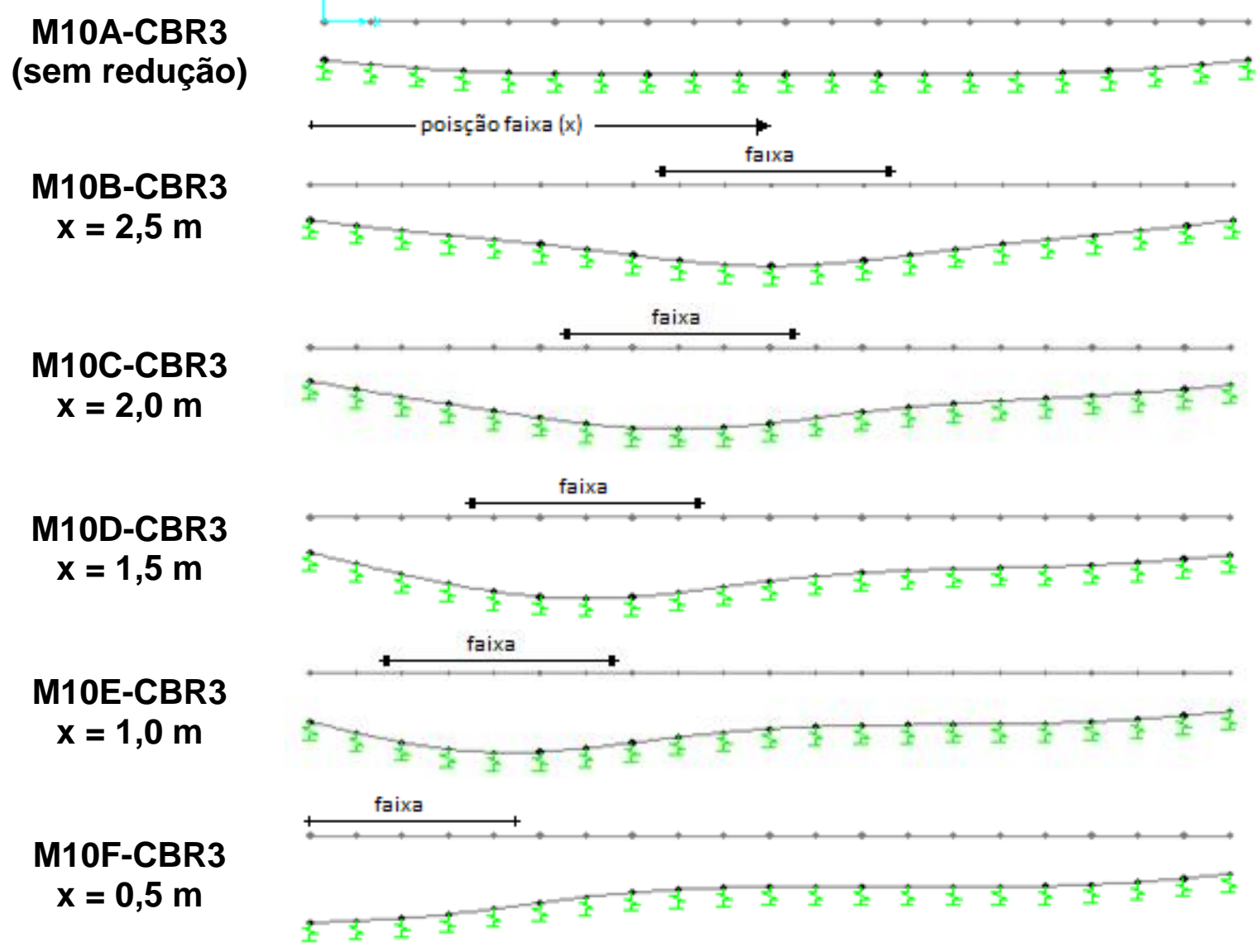

Figura 10: Deformações conforme posição da faixa reduzida, efeito de "onda". 
CAVIGLIONE, G.; REIS, J., ESTUDO DA INFLUÊNCIA DA COMPACTAÇÃO DE BASE NO SURGIMENTO DE TRINCAS EM PISOS INDUSTRIAIS DE CONCRETO CONSIDERANDO A INTERAÇÃO SOLO ESTRUTURA. $2^{\circ}$ Simpósio Paranaense de Patologia das Construções (2० SPPC), artigo 2SPPC2004, pp. 500-511, 2017. DOI: 10.4322/2SPPC.2017.041

Com os recalques obtidos pelo SAP, foi calculado o coeficiente de variação para cada modelo e situação, com isso é possível estimar qual seria a pior posição para a redução do módulo de resistência do solo. Um maior coeficiente de variação significa uma placa mais deformada, mais flexível, situação mais crítica. A Tabela 1 traz o resumo de cada situação, modelo e respectivo coeficiente de variação.

Tabela 1: Coeficientes de variação dos recalques.

\begin{tabular}{cccccccccc}
\hline CBR 3\% & M10 & M15 & M20 & M25 & CBR 10\% & M10 & M15 & M20 & M25 \\
\hline A & $25 \%$ & $21 \%$ & $17 \%$ & $13 \%$ & A & $26 \%$ & $23 \%$ & $20 \%$ & $17 \%$ \\
B & $25 \%$ & $21 \%$ & $17 \%$ & $13 \%$ & B & $27 \%$ & $23 \%$ & $19 \%$ & $16 \%$ \\
C & $25 \%$ & $21 \%$ & $17 \%$ & $13 \%$ & C & $27 \%$ & $23 \%$ & $19 \%$ & $16 \%$ \\
D & $24 \%$ & $18 \%$ & $15 \%$ & $13 \%$ & D & $26 \%$ & $21 \%$ & $17 \%$ & $15 \%$ \\
E & $20 \%$ & $16 \%$ & $15 \%$ & $14 \%$ & E & $24 \%$ & $18 \%$ & $16 \%$ & $15 \%$ \\
F & $24 \%$ & $23 \%$ & $22 \%$ & $21 \%$ & F & $25 \%$ & $23 \%$ & $22 \%$ & $22 \%$ \\
\hline
\end{tabular}

Observa-se (da tabela 1) que o comportamento se repete em todos os modelos, embora seja influenciado pela rigidez relativa. Para placas rígidas a pior localização para a redução é próxima à borda, ao passo que para placas flexíveis a pior posição situa-se entre "B" e "C" (2,5 m e 2,0 m medidos do início da viga). É possível notar que a posição "E" (1,0 m) é a menos desfavorável a placa.

Para avaliação da variação do recalque, chama-se de "recalque adicional" a diferença entre o recalque máximo e o recalque, no mesmo ponto, no modelo de controle. Foram obtidos os recalques adicionais para cada situação. Posteriormente, foi tomado o maior valor de recalque adicional para cada rigidez relativa, conforme equações 2, 3 e 5 (Figura 11a e 11b).

$\mathrm{Na}$ comparação com os momentos adicionais procedeu-se de maneira análoga. Os extremos do momento (máximos e mínimos) foram comparados de forma absoluta com os valores de momento no modelo de controle, no mesmo ponto. Foi registrado o maior valor de momento adicional para cada rigidez, comparando para todas as situações os momentos positivos e negativos (Figura 11c e 11d).

A)

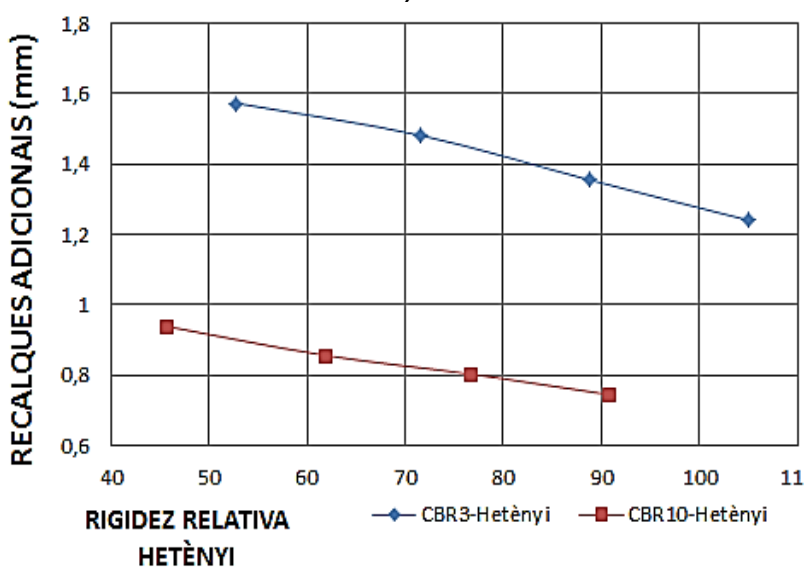

B)

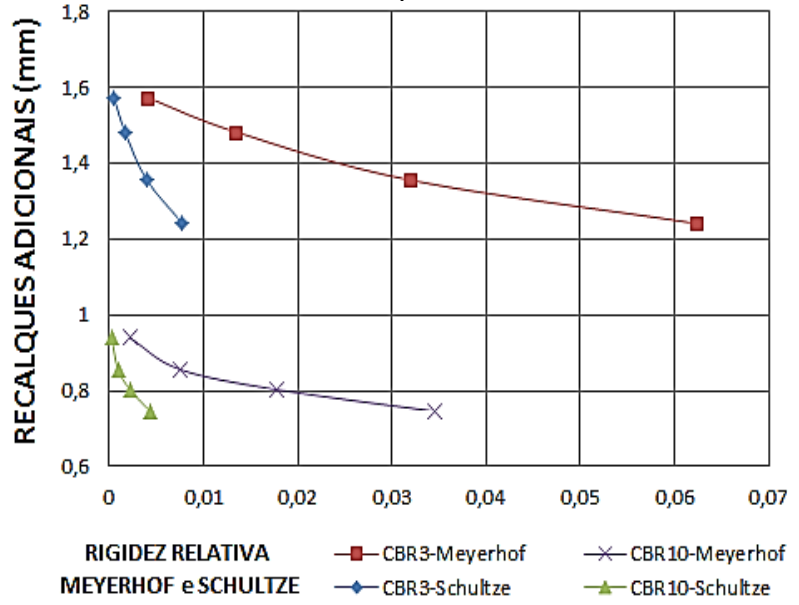


CAVIGLIONE, G.; REIS, J., ESTUDO DA INFLUÊNCIA DA COMPACTAÇÃO DE BASE NO SURGIMENTO DE TRINCAS EM PISOS INDUSTRIAIS DE CONCRETO CONSIDERANDO A INTERAÇÃO SOLO ESTRUTURA. $2^{\circ}$ Simpósio Paranaense de Patologia das Construções (20 SPPC), artigo 2SPPC2004, pp. 500-511, 2017. DOI: 10.4322/2SPPC.2017.041

C)

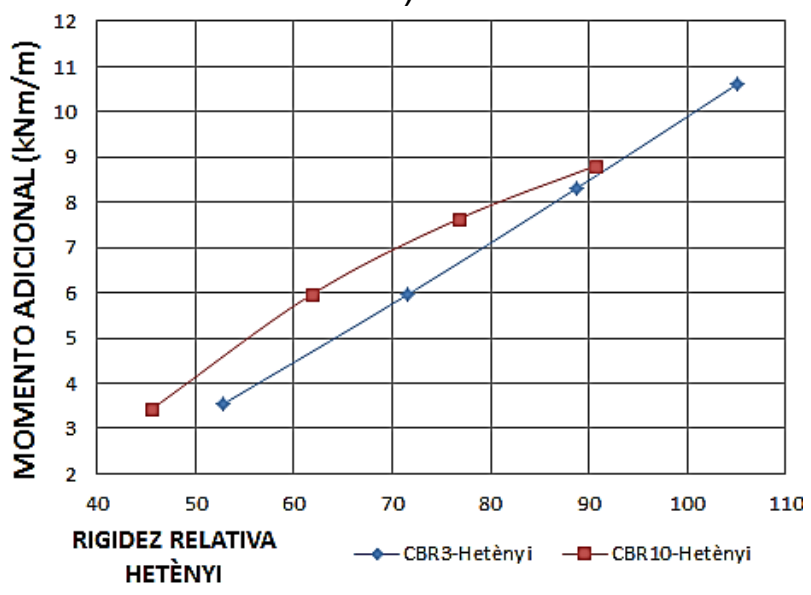

D)

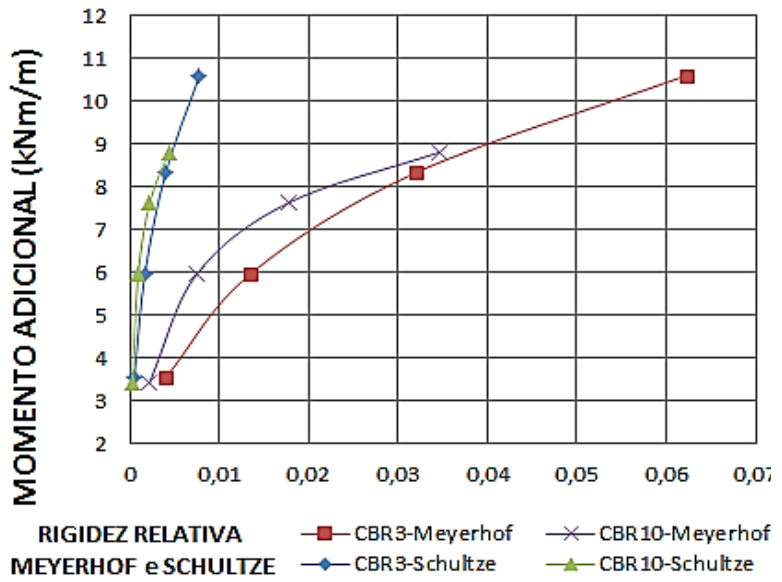

Figura 11: A) e B) Recalques adicionais e Rigidez relativa- C) e D) Momentos Fletores adicionais e Rigidez relativa.

Observa-se uma diminuição do recalque para aumento da rigidez relativa (Figura 11 a e Figura $11 b)$. Maiores valores de $K_{S S}$ representam estruturas mais rígidas em relação ao solo, com maior capacidade de distribuir as pressões no solo. Este comportamento repete-se em Hetènyi, Meyerhof e Schultze.

Os esforços solicitantes de flexão aumentam juntamente com a rigidez da estrutura, isto é, estruturas mais rígidas apresentaram maiores esforços (Figura 11c e 11d). Este comportamento era de se esperar em sistemas hiperestáticos, pois com 0 aumento da rigidez, a estrutura tem maior capacidade de distribuir esforços, deformando-se menos.

\section{Conclusões}

Nas placas mais rígidas a inversão dos esforços de flexão ocorre quando a faixa estava próxima à borda. Por isso, convém respeitar uma rigidez-relativa mínima a fim de evitar a inversão dos esforços de flexão. Embora se entenda que a compactação adequada é mais eficiente no combate ao problema.

A posição crítica para as placas depende da rigidez relativa. Para placas mais rígidas esta tende a aproximar-se da borda. Para placas flexíveis aproxima-se do centro. A posição menos crítica para compactação foi à distância de $1,0 \mathrm{~m}$ (1/5 do comprimento) da borda. O incremento de rigidez na estrutura provocou aumento nos esforços internos, e consequentemente redução dos recalques e valores de reação do solo, ratificando o modelo numérico.

A redução do módulo de reação foi tomada com valor de referência, mas tratandose da presença de água no solo, esse valor pode ser muito conservador. Em trabalhos futuros é interessante repetir o estudo com diferentes valores de $k_{v} \mathrm{e}$ carregamentos diferenciados. 


\section{Referências}

[1] Rodrigues, P. P. F; Botacini, S. M.; Gasparetto, W. E. (2006) Manual Gerdau de pisos industriais. Editora PINI, São Paulo, Brasil.

[2] Porto, T. B; Mendonça; B. Q.; Carvalho L. S. G. (2012) Análise estrutural de pisos industriais utilizando o método dos elementos finitos, Mecánica Computacional, 31:1355-1377.

[3] Cunha, P. F. (2013) Dimensionamento e análise numérica de pisos industriais de concreto. Dissertação (mestrado), Universidade Estadual do Rio de Janeiro - UERJ, Rio de Janeiro, Brasil.

[4] Rodrigues, P. P. F; Faria, B. M.; Silva, J. B. R. (2015) Pavimentos industriais de Concreto Armado. Instituto Brasileiro de Telas Soldadas - IBTS, São Paulo, Brasil.

[5] ANAPRE. (2009) Boletins Técnicos. Associação Nacional de Pisos e Revestimentos de Alto Desempenho - ANAPRE, São Paulo, Brasil.

[6] Velloso, D. A.; Lopes, F. R. (2010) Fundações. Oficina de textos, São Paulo, Brasil.

[7] Cintra, J. C. A.; Aoki, N.; Albiero, J.H. (2011) Fundações diretas: projeto geotécnico. Oficina de textos, São Paulo, Brasil.

[8] Souza, R. A.; Reis, J. H. (2008) Interação solo-estrutura para edifícios sobre fundações rasas, Acta Scientiarum Technology, 30:161-171.

[9] Dutta, S. C.; Roy, R. (2002) A critical review on idealization and modeling for interaction among soil-foundation-structure system, Computers and Structures, Elsevier, 80:1579-1594.

[10] Moraes, M. C. (1976) Estrutura de Fundações. McGraw-Hill e EDUSP, São Paulo, Brasil.

[11] Antoniazzi, J. P. (2011) Interação solo-estrutura de edifícios com fundações superficiais. Dissertação (mestrado), Universidade Federal de Santa Maria UFSM, Santa Maria, Brasil.

[12] Balbo, J. T. (2009) Pavimentos de concreto. Oficina de textos, São Paulo.

[13] Bowles, J. E. (1974) Analytical and Computer Methods in Foundation Engineering. McGraw-Hill, New York, Estados Unidos. 